\title{
Comparative Radiological Analysis of Efficacy of Beta-tricalcium Phosphate and Beta-tricalcium Phosphate with Platelet-rich Fibrin in Maxillary Sinus Augmentation - A Clinical Study
}

\author{
Srinivas Gadipelly, Salma Sultana, V. V. Venkatesh, P. Praveen \\ Department of Oral and Maxillofacial Surgery, Kamineni Institute of Dental Sciences, Narketpally, Nalgonda, Telangana State, India
}

Email for correspondence: salma.chammu89@gmail.com

\begin{abstract}
Context: Bone quantity in the maxillary posterior edentulous area may be insufficient for dental implant placement due to pneumatization of the maxillary sinus. Different solutions were proposed over the years, such as use of short implants, tilted implants placed in the anterior maxilla, zygoma implants, and maxillary sinus floor elevation and grafting procedures with autogenous bone or allografts, xenografts, and alloplastic materials. $\beta$-tricalcium phosphate ( $\beta$-TCP) is known to be excellent promotor of osteoblastic formation and readily resorbed by macrophages and osteoclasts and associated with a lower risk of failure or complications. The use of plateletrich fibrin $(\mathrm{PRF})$ during sinus lift procedures has been advocated for many years during lateral sinus lift or vertical osteotome augmentation. Aim: The aim of the present study was to evaluate the amount of bone formation, clinical and radiological outcomes, and post-operative morbidity of sinus floor elevation procedure performed using $\beta$-TCP and $\beta$-TCP in combination with PRF. Materials and Methods: Twenty patients who required bilateral sinus lift procedure were accepted into the study and were treated with sinus lift with $\beta$-TCP with platelet-rich fibrin (PRF) on one side (Group A) and with only $\beta$-TCP on the other side (Group B). The bone height of the ridges was measured on computed tomography (CT) scans (Dentascans) of $0.6 \mathrm{~mm}$ sections. Vertical bone heights of all the patients were measured preoperatively and postoperatively on CT scans and the amount of vertical bone gain was assessed. Results: The mean bone height of all the patients who were treated with a direct sinus lift (preoperatively) was $6.0 \mathrm{~mm}$ in Group A and $5.8 \mathrm{~mm}$ in Group B and the heights varied anywhere between $3.5 \mathrm{~mm}$ and $7.4 \mathrm{~mm}$ for a total of 50 implant sites. The mean bone gain in direct sinus lift was $5.91 \mathrm{~mm}$ on the side of $\beta$-TCP with PRF and $5.01 \mathrm{~mm}$ on the side of $\beta$-TCP. Bone density between the two groups showed statistically significant difference at the $6^{\text {th }}$ month recall when measured by independent $t$-test. Conclusion: The study indicated that $\beta$-TCP with PRF and $\beta$-TCP is both effective bone substitutes for sinus floor elevation in oral and maxillofacial region. The mean bone gain in direct sinus lift on $\beta$-TCP is comparatively less than $\beta$-TCP with PRF.
\end{abstract}

Key words: Bone gain, lateral approach, platelet-rich fibrin, sinus lift, $\beta$-tricalcium phosphate

\begin{tabular}{l|l|}
\hline Quick Response Code & Article Info: \\
\hline doi: 10.5866/2018.10.10171 \\
\hline $\begin{array}{l}\text { Received: } 24-09-2018 \\
\text { Revised: } 22-10-2018 \\
\text { Accepted: } 19-11-2018 \\
\text { Available Online: } 05-01-2019, \text { (www. } \\
\text { nacd.in)@ NAD, 2019 - All rights reserved }\end{array}$ \\
\hline
\end{tabular}

\section{INTRODUCTION}

The maxillary posterior edentulous area presents a difficult situation in implantology when compared to other areas of mouth. Bone quantity in the maxillary posterior edentulous area may be insufficient for dental implant placement due to the presence of the maxillary sinus. When teeth are extracted in the posterior maxilla, bone in that 
area is lost due to inferior expansion of the sinus involving the residual ridge area. This process is known as pneumatization of the maxillary sinus. ${ }^{[1]}$ Pneumatization seems to be due to bone loss, which is caused by increased osteoclastic activity of the sinus membrane. Sufficient bone quantity and quality is required for dental implant placement. To overcome these problems, different solutions were proposed over the years, such as use of short implants, tilted implants placed in the anterior maxilla, zygoma implants, and maxillary sinus floor elevation and grafting procedures with autogenous bone or allografts, xenografts, and alloplastic materials. ${ }^{[2]}$ The antral membrane balloon elevation technique is another minimally invasive technique to elevate the sinus membrane. ${ }^{[3]}$ Many clinicians believe that more traditional sinus lift procedures, such as a lateral approach, should be used instead of subantral augmentation, if the alveolar bone height is to be raised $>3 \mathrm{~mm}$, although surface-enhanced dental implants can often compensate for minimal bone height in the augmented site. ${ }^{[4]}$ The lateral approach using a Caldwell-Luc osteotomy is historically the first main technique, where the maxillary sinus floor is grafted to provide a sufficient quantity of bone for the placement of endosteal dental implants.

In sinus lift procedure, the approach to increase bone availability for placing implants uses a "window" in the lateral bony wall of the maxillary sinus to gain access to the underlying Schneiderian membrane. ${ }^{[5]}$ Using this approach, implant placement can be performed in one or two surgical stages depending on the residual alveolar bone height. Various types of biomaterials can be prepared in the granular form including morcellized autograft, cleaned and purified allo- (or) xeno-genic bone, natural biomaterials, and pure synthetic calcium phosphate biomaterials ( $\beta$-tricalcium phosphate $[\beta-T C P]$ hydroxyapatite). Sinus lift elevation restores bone mass at the maxilla in edentulous patients before the placement of dental implants. It consists of opening the lateral side of the sinus and grafting $\beta$-TCP granules.

$\beta$-TCP is known to be excellent promotor of osteoblastic formation and readily resorbed by macrophages and osteoclasts. $\beta$-TCP granules were prepared by the polyurethane foam technology. ${ }^{[6]}$ Nyangoga et al. reported-TCP to be associated with a lower (or the same) risk of failure or complication. ${ }^{[7]}$ It is known that resorption of $\beta$-TCP depends on the amount of implanted and the patient's age.
The mechanism of bioceramic resorption involves two processes, solution mediate disintegration and cell-mediated disintegration. ${ }^{[7]}$ The use of plateletrich fibrin (PRF) during sinus lift procedures has been advocated for many years during lateral sinus lift or vertical osteotome augmentation. ${ }^{[8]}$ With this background, the present study was aimed to compare and evaluate the alveolar bone growth on maxillary sinus after bilateral sinus lift procedures, performed using $\beta$-TCP with PRF (Group-A) and $\beta$-TCP (Group-B) alone with saline on the other side, with the simultaneous placement of dental implants.

\section{MATERIALS AND METHODS}

A total of 20 patients from the OPD of Kamineni Institute of Dental Sciences, Narketpally, who needed implants in the maxillary posterior region with deficient maxillary ridge height were selected for the present study [Figure 1]. Patients are divided into Group A and Group B. In Group A, 10 patients were selected for $\beta$-TCP with PRF, and in Group B, the other 10 were selected for $\beta$-TCP without PRF.

\section{Inclusion Criteria}

Patients included in the study were, partially edentulous jaws with unilateral or bilateral loss of teeth in maxillary premolar or molar area, residual alveolar ridge height of $8 \mathrm{~mm}$ or less with no buccolingual augmentation.

\section{Exclusion Criteria}

Patients with uncontrolled diabetes, autoimmune diseases, and patients undergoing radiation or chemotherapy to head and neck region were excluded from the study.
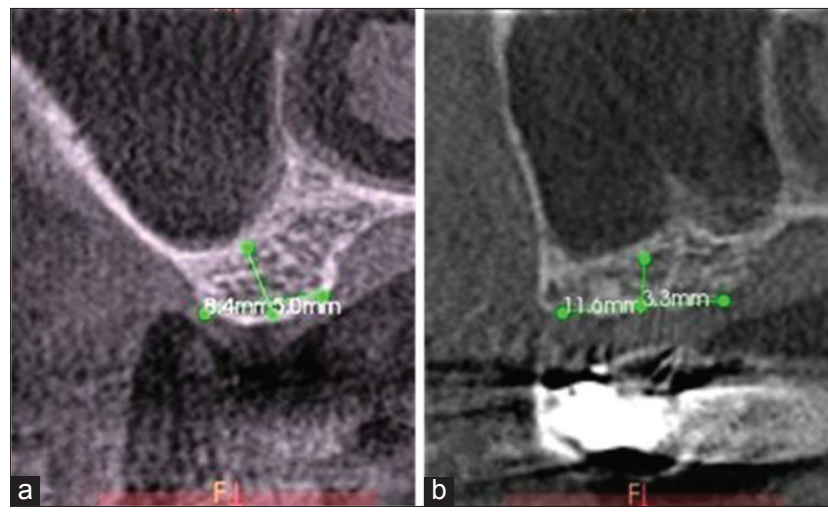

Figure 1: (a) Pre-operative computed tomography (CT) scan shows insufficient height of bone $(5.0 \mathrm{~mm})$ and was selected under Group A; (b) Pre-operative CT scan shows insufficient height of bone $(3.3 \mathrm{~mm})$ and was selected under Group B 


\section{Methodology}

Patient was evaluated by the following methods:

I. Clinical evaluation of the implant.

II. To evaluate the implant and bone interface on periapical radiographs, taken after 6 months.

III. Evaluation of the amount of bone formation post-surgery on orthopantomogram (OPG) taken at 6 months.

IV. To evaluate by means of computerized tomographic scans,

a) Evaluation of the amount of bone gain obtained post-surgery on computed tomography (CT) scan taken at 6 months.

b) The bone remodeling around implants placed in the reconstructed region.

\section{Procedure}

\section{PRF preparation}

Around $5 \mathrm{ml}$ of whole venous blood was withdrawn and collected in one $10 \mathrm{ml}$ sterile test tube or $10 \mathrm{ml}$ of whole venous blood was withdrawn and collected equally ( $5 \mathrm{ml}$ each) in two sterile glass tubes of $6 \mathrm{ml}$ capacity without of anticoagulant depending on the number of sites. The test tubes were then placed in a centrifugal machine at 3000 RPM for $10 \mathrm{~min}$, after which it settled into the following layers: Red lower fraction, the fibrin clot containing red blood cells, upper straw-colored cellular plasma and middle fraction containing the fibrin clot. The upper straw-colored layer was removed using pipette and middle fraction was collected, $2 \mathrm{~mm}$ below lower dividing line, which is the PRF. This PRF clot was then used as a filling material for sinus floor augmentation. Sinus membrane elevation defects were then filled with $\beta$-TCP on one side with PRF (Group-A) on one side and alone $\beta$-TCP (Group-B) on the other. The graft was mixed with PRF on one side and with normal saline on other side, and then, the medial aspect of the sinus cavity was filled. Immediate implant placement was done.

\section{Sinus Lift Procedure}

The buccal window technique was used when the bone height was $>6 \mathrm{~mm}$ between the crest of the ridge and the floor of the maxillary sinus. A local anesthetic, with a vasoconstrictor for hemostasis, was infiltrated into maxillary surgical site. The surgery was performed under local anesthesia. Posterior superior alveolar and greater palatine nerve blocks combined with infiltrations were given. A horizontal incision was made on the crest or palatal aspect of edentulous ridge, with extensions beyond the areas of the osteotomy and with consideration of the amount of attached gingiva on the alveolar crest. The incision was carried forward beyond the anterior border of the sinus. A vertical releasing incision was given in the canine fossa. The lateral wall of the maxilla was exposed by reflecting the mucoperiosteal flap superiorly to the level of the malar buttress [Figure 2]. Elevation of the periosteum adjacent to the implant site was minimized to preserve the blood supply to the alveolar crest. The periosteum was reflected superiorly, just beyond the height of the superior aspect of the anticipated opening into the maxillary sinus. Sinus floor elevation is performed with $\beta$-TCP with PRF [Figure 3]. Closure done with 3-0 vicryl [Figure 4] and immediate implant placement was done.

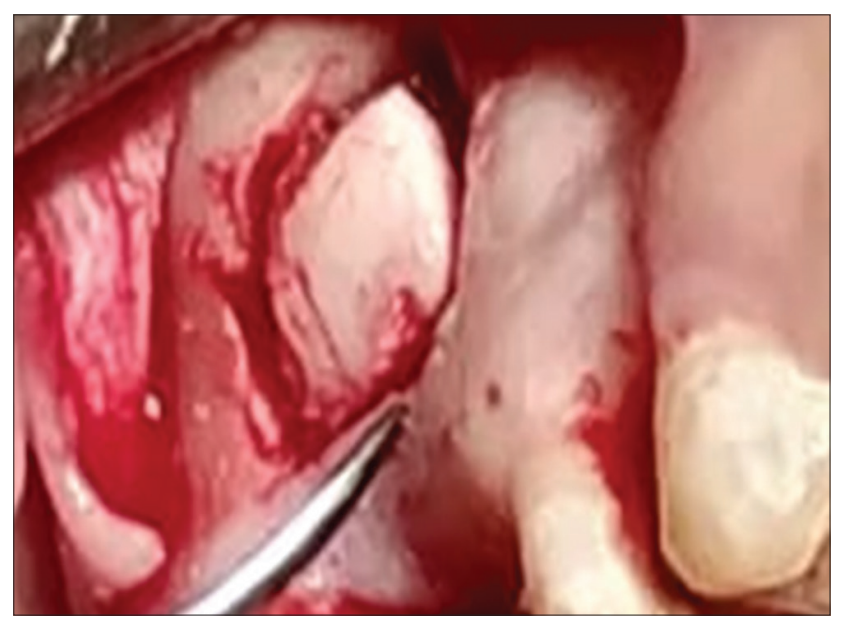

Figure 2: Clinical picture showing lateral window preparation

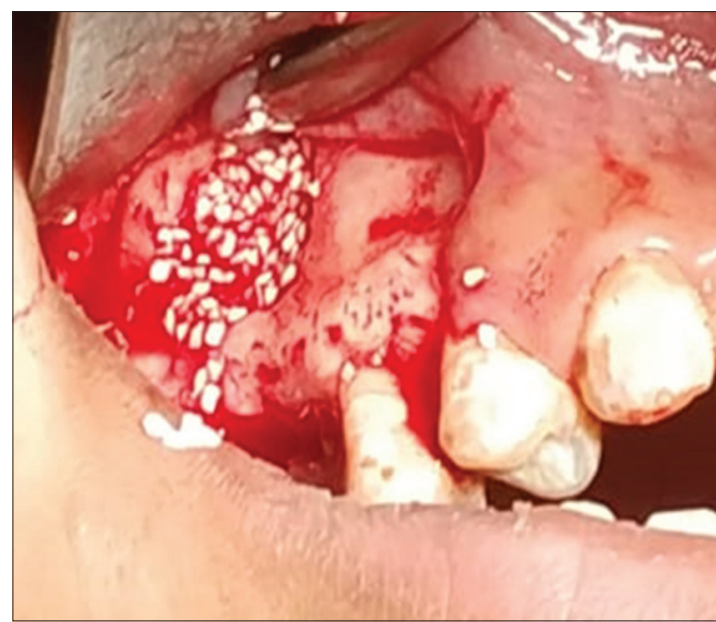

Figure 3: Clinical picture showing the placement of bone graft $-\beta$-tricalcium phosphate with platelet-rich fibrin 


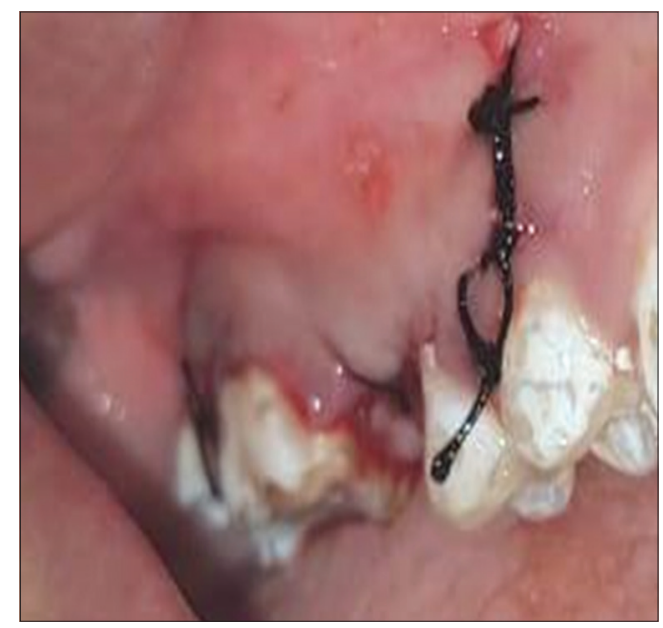

Figure 4: Clinical picture showing closure done with 3-0 vicryl

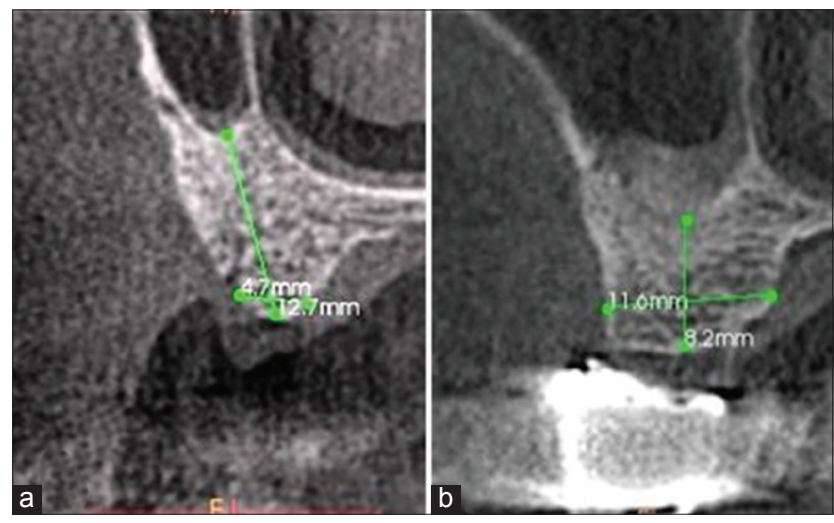

Figure 5: (a) Post-operative computed tomography (CT) scan shows increased height of bone $(12.7 \mathrm{~mm})$ in Group A treated with $\beta$-tricalcium phosphate ( $\beta$-TCP) and platelet-rich fibrin;

(b) post-operative CT scan shows increased height of bone $(8.2 \mathrm{~mm})$ in Group B treated with $\beta$-TCP alone

\section{Follow-up}

Postoperatively, bone height and density were measured, which were performed after 1 week and 6 months after surgery, and the lifted maxillary sinus was clinically checked for sinus infections. Patients were followed for 6 months after abutment connection.

\section{RESULTS}

An independent sample " $t$ " test has been applied to assess the difference between $\beta$-TCP and PRF, and $\beta$-TCP applied sides and in terms of the outcome, i.e., the bone density in CT was measured by Hounsfield units at the $6^{\text {th }}$ month

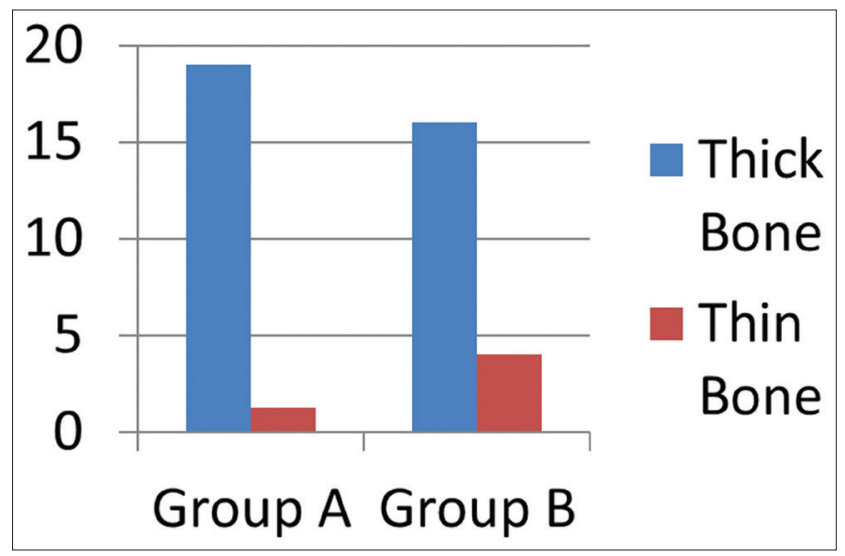

Graph 1: Regenerated bone on computed tomography in Groups A and B

recall. A significant increase in height of bone was observed in both the groups, where Group A has more increase in bone height when compared to Group B [Figure 5].

Independent sample " $t$ " test: $\beta$-TCP with PRF showed significant difference at the $6^{\text {th }}$ month recall (as suggested by mean bone density values) in bone density when compared to $\beta$-TCP alone in buccolingual, axial, and panoramic projections $(P<0.05)$ and overall mean density scores were also significantly improved in the side of $\beta$-TCP and PRF as compared to the side of $\beta$-TCP.

\section{DISCUSSION}

In the present study, the alveolar bone growth was compared between the two sides of the maxillary sinus after bilateral sinus lift procedures performed with the simultaneous placement of dental implants in 20 consecutive patients. The amount of bone gain was evaluated both in the OPGs and CT scans. While the results in the OPG are not predictable, so more emphasis was given on the CT scans.

In the present study, in one case, the residual alveolar height was $5.4 \mathrm{~mm}$ in Group A preoperatively [Figure 1a] and $3.3 \mathrm{~mm}$ in Group B [Figure 1b] and the bone gain achieved on the $\beta$-TCP with PRF (Group-A) side was $12.7 \mathrm{~mm}$ [Figure 5a] and $8.2 \mathrm{~mm}$ on the side, of which $\beta$-TCP (Group-B) alone [Figure $5 \mathrm{~b}$ ] was used, showing the superiority of PRF as a viable healing tissues. The same was seen in all the other cases too.

On the $\beta$-TCP with PRF side, the regenerated bone was more thicker than that of the native bone and it was statistically significant in 19 cases and less than native bone in one case. Whereas 
on the side $\beta$-TCP alone was used, the bone was more thicker in 16 cases which was statistically significant and less than (thinner) that of the native bone in four cases [Graph 1].

Meyer et al. evaluated the long-term reliability of pure phase $\beta$-TCP and concluded that the implant success rate was $97.6 \%$ (lack of osteointegration for two implants in the same patient, one failure after loading) ${ }^{[9]}$ In the present study also failure rate was less, where only one implant failure was seen in Group A and two implant failures were seen in Group B; and the reason for failure is not known.

Elmohandes evaluated the augmentation of atrophic maxillary bone using $\beta$-TCP granules mixed with PRF for sinus lift and then implant installation 4 months later. They concluded that the implants showed good stability during installation as well as at 12 months afterinstallation, and also, the study showed consistent bone formation at the maxillary sinus floor following the use of mixture of both $\beta$-TCP granules and PRF as well as after implant installation. ${ }^{[10]}$ The present study is also in concordance with Elmohandes findings where superior bone formation was seen at the maxillary sinus floor following the use of mixture of both $\beta$-TCP granules and PRF as well as after implant installation.

\section{CONCLUSION}

The present study suggests that the use of the $\beta$-TCP and PRF mixture can reduce long time needed for new bone formation, as well as the morbidity risk related to harvesting autogenous bone graft. This result seems to indicate that a higher percentage of new bone formation occurs in the side where PRF was used because no resorption of graft material is required and the morphological assessment on CT scans has shown that $\beta$-TCP with $\mathrm{PRF}$ is superior to $\beta$-TCP alone.

\section{REFERENCES}

1. Abdallah J. Augmentation procedures for the maxillary sinus: A case report with 10-year follow-up. Int J Oral Implant Clin Res 2011;2:105-9.

2. Rodríguez-Chessa JG, Olate S, Netto HD, Shibli J, de Moraes M, Mazzonetto R. Treatment of atrophic maxilla with zygomatic implants in 29 consecutives patients. Int $\mathrm{J}$ Clin Exp Med 2014;7:426-30.

3. Tarun Kumar AB, Anand U. Maxillary sinus augmentation. J Int Clin Dent Res Organ 2015;7:81-93.

4. Steigmann M, Garg AK. A comparative study of bilateral sinus lifts performed with platelet-rich plasma alone versus alloplastic graft material reconstituted with blood. Implant Dent 2005;14:261-6.

5. Toscano NJ, Holtzclaw D, Rosen PS. The effect of piezoelectric use on open sinus lift perforation: A retrospective evaluation of 56 consecutively treated cases from private practices. J Periodontol 2010;81:167-71.

6. Peleg M, Garg AK, Mazor Z. Predictability of simultaneous implant placement in the severely atrophic posterior maxilla: A 9-year longitudinal experience study of 2132 implants placed into 731 human sinus grafts. Int J Oral Maxillofac Implants 2006;21:94-102.

7. Nyangoga H, Aguado E, Goyenvalle E, Baslé MF, Chappard D. A non steroidal anti inflammatory drug (ketoprofen) does not delay $\beta$-TCP bone graft healing. J Acta Biomater 2010;6:33107.

8. Tanaka T, Kumagae Y, Saito M, Chazono M, Komaki H, Kikuchi $\mathrm{T}$, et al. Bone formation and resorption in patients after implantation of beta-tricalcium phosphate blocks with $60 \%$ and $75 \%$ porosity in opening-wedge high tibial osteotomy. J Biomed Mater Res B Appl Biomater 2008;86:453-9

9. Meyer C, Chatelain B, Benarroch M, Garnier JF, Ricbourg B, Camponovo T. Massive sinus-lift procedures with betatricalcium phosphate: Long-term results. Rev Stomatol Chir Maxillofac 2009;110:69-75.

10. Elmohandes W. Evaluation of beta tricalcium phosphate mixed with platelet rich fibrin for rehabilitation of atrophic maxilla with implant installation. Int $\mathrm{J}$ Oral Maxillofac Surg 2013;7:303. 